\title{
Long-term total sleep deprivation decreases the default spontaneous activity and connectivity pattern in healthy male subjects: a resting-state fMRI study
}

\author{
This article was published in the following Dove Press journal: \\ Neuropsychiatric Disease and Treatment \\ 19 March 2015 \\ Number of times this article has been viewed
}

\begin{abstract}
Xi-Jian Dai, 1,2,* Chun-Lei Liu, ${ }^{3,4, *}$ Ren-Lai Zhou ${ }^{3}$ HongHan Gong,' Bin Wu, ${ }^{5}$ Lei Gao,' Yi-Xiang J Wang ${ }^{2}$

'Department of Radiology, The First Affiliated Hospital of Nanchang University, Nanchang, Jiangxi, People's Republic of China; ${ }^{2}$ Department of Imaging and Interventional Radiology, Prince of Wales Hospital, The Chinese University of Hong Kong, Shatin, New Territories, Hong Kong SAR, People's Republic of China; ${ }^{3}$ Beijing Key Lab of Applied Experimental Psychology, School of Psychology, and National Key Laboratory of Cognitive Neuroscience and Learning, Beijing Normal University, Beijing, People's Republic of China; ${ }^{4}$ School of Education, Qufu Normal University, Qufu, Shandong, People's Republic of China; ${ }^{5}$ National Key Laboratory of Human Factors Engineering, China Astronaut Research and Training Center, Beijing, People's Republic of China

*These authors have contributed equally to this work
\end{abstract}

Correspondence: Ren-Lai Zhou Beijing Key Lab of Applied Experimental Psychology, School of Psychology, and National Key Laboratory of Cognitive Neuroscience and Learning, Beijing

Normal University, Beijing 100875 ,

People's Republic of China

Tel +86 0105880202

Email rlzhou@bnu.edu.cn

Hong-Han Gong

Department of Radiology, The First

Affiliated Hospital of Nanchang University,

Number 17, YongWai Zheng Street,

DongHu District, Nanchang 330006 ,

Jiangxi, People's Republic of China

$\mathrm{Tel}+86$ 79| 88692582

Email honghan_gong@sina.com
Objective: The aim of this study is to use resting-state functional connectivity (rsFC) and amplitude of low-frequency fluctuation (ALFF) methods to explore intrinsic default-mode network (DMN) impairment after sleep deprivation (SD) and its relationships with clinical features.

Methods: Twelve healthy male subjects underwent resting-state functional magnetic resonance imaging twice: once following rested wakefulness (RW) and the other following 72 hours of total SD. Before the scans, all subjects underwent the attention network test (ANT). The independent component analysis (ICA), rsFC, and ALFF methods were used to examine intrinsic DMN impairment. Receiver operating characteristic (ROC) curve was used to distinguish SD status from RW status.

Results: Compared with RW subjects, SD subjects showed a lower accuracy rate $(\mathrm{RW}=96.83 \%$, $\mathrm{SD}=77.67 \% ; P<0.001)$, a slower reaction time ( $\mathrm{RW}=695.92 \mathrm{~ms} ; \mathrm{SD}=799.18 \mathrm{~ms} ; P=0.003)$, a higher lapse rate $(\mathrm{RW}=0.69 \%, \mathrm{SD}=19.29 \% ; P<0.001)$, and a higher intraindividual coefficient of variability in reaction time $(\mathrm{RW}=0.26, \mathrm{SD}=0.33 ; P=0.021)$. The ICA method showed that, compared with RW subjects, SD subjects had decreased rsFC in the right inferior parietal lobule (IPL, BA40) and in the left precuneus (PrC)/posterior cingulate cortex (PCC) (BA30, 31). The two different areas were selected as regions of interest (ROIs) for future rsFC analysis. Compared with the same in RW subjects, in SD subjects, the right IPL showed decreased rsFC with the left PrC (BA7) and increased rsFC with the left fusiform gyrus (BA37) and the left cluster of middle temporal gyrus and inferior temporal gyrus (BA37). However, the left $\operatorname{PrC}$ / PCC did not show any connectivity differences. Compared with RW subjects, SD subjects showed lower ALFF area in the left IPL (BA39, 40). The left IPL, as an ROI, showed decreased rsFC with the right cluster of IPL and superior temporal gyrus (BA39, 40). ROC curve analysis showed that the area under the curve (AUC) value of the left IPL was 0.75 , with a cutoff point of 0.834 (mean ALFF signal value). Further diagnostic analysis exhibited that the AUC alone discriminated SD status from RW status, with 75\% sensitivity and $91.7 \%$ specificity.

Conclusion: Long-term SD disturbed the spontaneous activity and connectivity pattern of DMN.

Keywords: sleep deprivation, amplitude of low-frequency fluctuation, default-mode network, functional magnetic resonance imaging, functional connectivity, independent component analysis, receiver operating characteristic curve

\section{Introduction}

Sleep deprivation (SD) is common and can be a serious problem negatively affecting health. Sleep has been reported to allow the removal of free radicals accumulated 
in the brain during wakefulness, ${ }^{1}$ while SD can induce oxidative damage and disturb the removal of free radicals in the brain. ${ }^{2}$ Long-term SD is harmful to health and can lead to multisystemic and multiorgan dysfunction, causing maladaptive emotional regulation, exaggerated neural reactivity, aversive experiences, and negative metabolic, psychological, physiological, or even behavioral reactivity. ${ }^{3-6}$ However, their mechanisms remain unclear.

Anatomically the "default-mode network" (DMN) spans the bilateral inferior parietal lobule (IPL), posterior cingulate cortex (PCC), precuneus (PrC), medial prefrontal cortex (MPFC), retrosplenial cortex, and parts of the hippocampal formation and medial temporal lobe. ${ }^{7,8}$ Recent research has suggested that the DMN might be associated with collection and evaluation of information, ${ }^{9}$ self-referential mental activity, ${ }^{10}$ extraction of episodic memory, ${ }^{11}$ emotion and anxiety, ${ }^{12,13}$ and mind wandering or daydreaming. ${ }^{14}$ More and more researches have been devoted to exploring the DMN changes in various kinds of diseases, including cognitive impairment, ${ }^{15,16}$ chronic pain, ${ }^{17}$ hepatic encephalopathy, ${ }^{18}$ and autism. ${ }^{19}$ Furthermore, the focus of many researches has been on delineating the regional effects of SD on DMN. Gujar et $\mathrm{al}^{20}$ found that deactivation within DMN regions alone discriminated SD from sleep-control subjects with $93 \%$ sensitivity and $92 \%$ specificity. In addition, the relative balance of deactivation within these default nodes significantly correlated with the amount of prior sleep in the control group. Bosch et $\mathrm{al}^{21}$ found that $\mathrm{SD}$ reduced resting-state functional connectivity (rsFC) between PCC and bilateral anterior cingulate cortex, in addition to enhancing rsFC between the dorsal nexus and distinct areas in the right dorsolateral PFC. Chee and $\mathrm{Chuah}^{22}$ found SD-related reduced IPL deactivation during a visual short-term memory task. Horovitz et $\mathrm{al}^{23,24}$ revealed reductions in the IPL-MPFC rsFC during deep sleep and after partial SD. De Havas et $\mathrm{al}^{25}$ also found that the IPL node of the DMN was consistently impaired and might represent an early marker for the effects of 24-hour $\mathrm{SD}$, as well as serving as an indicator of hitherto-unexplored behavioral impairments. Although these studies provide insights into the neural events occurring during SD, far less is still known about the effect of long-term SD on intrinsic DMN node impairment.

It is proposed that resting-state functional magnetic resonance imaging (rfMRI), one of the hot areas in neuroimaging and one that is suitable for the mechanism research of central nervous system, can detect the spontaneous neuronal activity of the human brain and provide new insights into the pathophysiology of disease, because of its advantages in not requiring exposure to radioactive tracers, accurate positioning, and ease of combining functional imaging with structural imaging. Amplitude of low-frequency fluctuation (ALFF) is an index in which the square root of the power spectrum is integrated in a low-frequency range for detecting the regional spontaneous neuronal activity in blood oxygenation-level dependent (BOLD) signal. ${ }^{26}$ Previous studies have demonstrated that the ALFF showed good-to-moderate test-retest reliability. ${ }^{27,28}$ The simple calculation and reliable characterization of the ALFF measurement makes it a suitable and useful tool for rfMRI data analysis to investigate a disease trait. The ALFF measurement has been successfully applied in the exploration of functional modulations and characterization of the pathophysiological changes during different sleep conditions, such as the condition of being awake ${ }^{29}$ and light sleep. ${ }^{30}$ However, little attention has been given to the possibility that SD may be equally well described on the basis of differences in resting-state modes of spontaneous brain activation. The rsFC indicates temporal correlations among neural or hemodynamic signals arising from distinct brain regions, and it measures the signal synchrony of ALFF activity among different brain areas, while it still cannot ascertain which brain area is abnormal when abnormal rsFC is seen between two or more areas. Thus, it is desirable that the ALFF and the rsFC methods are used together.

In this study, we hypothesized that long-term SD disturbs spontaneous default activity and connectivity pattern. For a more comprehensive understanding of the effect from long-term total SD on DMN, we examined the statistical differences between long-term total SD condition and rested wakefulness (RW) condition, both in regional spontaneous activities using the ALFF method from regional perspectives and in default connectivity pattern using the rsFC method from integrative perspectives.

\section{Materials and methods \\ Subjects}

The sleeping habit and sleep quality of all participants were monitored throughout 1-week duration by wearing a Fitbit Flex tracker (http://help.fitbit.com/), and only those whose data indicated habitual good sleep were recruited for this study. Twelve healthy male participants, aged 20-32 years (mean: $24.83 \pm 2.88$ years) with an education duration of $15.6 \pm 1.5$ years and who were university students responding to a Web-based questionnaire, were recruited. All subjects met the following criteria as in our previous studies ${ }^{3,31}: 1$ ) had no symptoms associated with sleep disorders and no history of any psychiatric or neurologic disorders; 2) right-handed; 
3) had a good sleeping habit, ie, $>6.5$ hours of sleep per night, falling asleep no later than 1.00 am and waking up no later than $9.00 \mathrm{am}$; 4) had a good sleep onset and/or maintenance, and has no history of swing shift, shift work, or sleep complaints; 5) had a regular dietary habit and did not consume any stimulants, alcohol, tea, cigarette, medications, and caffeine for at least 3 months before the study; 6) had no foreign implants in the body and no inborn or other acquired diseases; 7) moderate body shape and weight; and 8) Pittsburgh Sleep Quality Index score $<5$, and Hamilton Depression Rating Scale and Hamilton Anxiety Rating Scale scores $<7$. The protocols conformed to the Declaration of Helsinki and were approved by the First Affiliated Hospital of Nanchang University. All participants participated voluntarily and gave their written informed consent.

\section{Sleep deprivation}

The experiment was conducted in a closed narrow room, which was about $8 \mathrm{~m}^{2}$ large and was equipped with monitors and ventilation. Two doors were used to isolate the room and the supervisory room. The researchers did not interact directly with the participants during the SD experiment. Thus, food and other necessities were passed through the doors. The SD was from 9.00 am of the first day to 9.00 am on the third day. During the 72 hours of total SD, the participants were required to stay awake all the time. The staffs of the research team took charge of monitoring in turns through video, preventing participants from falling asleep. If there were any signs of falling asleep, they were awakened by an alarm clock immediately.

\section{Research design and process}

Each of the subjects underwent rfMRI scans twice at 9:15 am; one followed RW and the other followed 72 hours of total SD. During the rfMRI scans, the subjects were asked to look at the black screen in the MRI scanner, where a "+" still stay on the screen. A video monitor was installed to ensure that the participants were awake with their eyes open during the rfMRI scans. A simple questionnaire was administered immediately to inquire whether the subjects were awake after the scans. Before the scans, all subjects underwent the attention network test (ANT). ${ }^{32,33}$

\section{Image acquisition}

MRI scanning was performed ona3-TeslaMRI scanner(Trio; Siemens, Erlangen, Germany). Each subject underwent a 7-minute fMRI scan immediately after T1-weighted images were obtained. High-resolution T1-weighted images were acquired with a three-dimensional spoiled gradient-recalled sequence in a sagittal orientation: 176 images (repetition time $=2,530 \mathrm{~ms}$, echo time $=3.39 \mathrm{~ms}$, thickness $=1.33 \mathrm{~mm}$, gap $=0 \mathrm{~mm}$, matrix $=256 \times 256$, field of view $=240 \mathrm{~mm} \times 240 \mathrm{~mm}$, flip angle $=7^{\circ}$ ) were obtained. Finally, the 210 functional images (repetition time $=2,000 \mathrm{~ms}$, echo time $=30 \mathrm{~ms}$, thickness $=3.5 \mathrm{~mm}$, gap $=0.7 \mathrm{~mm}$, matrix $=64 \times 64$, flip angle $=90^{\circ}$, field of view $=220 \mathrm{~mm} \times 220 \mathrm{~mm}, 33$ axial slices with gradientrecalled echo-planar imaging pulse sequence) covering the whole brain were obtained.

\section{Data analysis}

\section{Behavioral data analysis}

On the basis of the ANT, the behavioral accuracy rate was measured using corrected recognition; the reaction time (RT) was determined using only trials with correct responses (correct RT), and the lapse rate was measured using missing recognition. The intraindividual coefficient of variation was calculated for each participant by dividing the standard deviation of the correct RT by the mean correct RT.

\section{fMRI data analysis}

Data preprocessing

Functional data were checked by MRIcro software (www.MRIcro.com) to exclude defective data. The first ten time points of the functional images were discarded due to the possible instability of the initial MRI signal and the participants' adaptation to the scanning environment. Using MATLAB2010a (Mathworks, Natick, MA, USA), the remainder of the data preprocessing was performed by DPARSFA (http://rfmri.org/DPARSF) software, including Digital Imaging and Communications in Medicine standards for form transformation, slice timing, head motion correction, spatial normalization, and smoothing with a Gaussian kernel of $6 \times 6 \times 6 \mathrm{~mm}^{3}$ full-width at half-maximum. Motion time courses were obtained by estimating the values for translation (millimeters) and rotation (degrees) for each subject. Participants who had $>2.0-\mathrm{mm}$ maximum displacement in the $x, y$, or $z$ directions and $2.0^{\circ}$ of angular motion during the whole rfMRI scans were rejected. The Friston six head motion parameters were used to regress out head motion effects based on recent work showing that higher-order models were more effective in removing head motion effects. ${ }^{34,35}$ Linear regression was also applied to remove other sources of spurious covariates along with their temporal derivatives, including the signal from a ventricular region of interest (ROI) and the signal from a region centered in the white matter. ${ }^{36}$ Of note, the global signal was not regressed out in 
the present data, as in our previous study, ${ }^{31}$ for the reason that it is still controversial to remove the global signal in the preprocessing of resting-state data. ${ }^{36,37}$ After head motion correction, the fMRI images were spatially normalized to the Montreal Neurological Institute space using the standard echo-planar imaging template and resampling the images at a resolution of $3 \mathrm{~mm} \times 3 \mathrm{~mm} \times 3 \mathrm{~mm}$.

\section{Independent component analysis}

After data preprocessing, the smoothed images of each subject were inserted into an overall independent component analysis (ICA) to decompose fMRI data into spatially independent component (IC) patterns and time courses by using the group ICA of fMRI toolbox (GIFT). ${ }^{38}$ The fMRI data were split into a set of spatially independent functional networks (components). Each component was presented as a spatial map along with an associated time course. The optimal number of ICs was estimated using a modified minimum description length algorithm, ${ }^{39}$ and this number was found to be 22 in this study. According to the templates provided by GIFT, the corresponding ICs of the DMNs for each subject were individualized and selected. The details of ICA analysis were reported in a previous study. ${ }^{40}$

\section{ALFF differences}

After preprocessing, the time series for each voxel were temporally bandpass filtered $(0.01-0.08 \mathrm{~Hz})$ and linearly detrended to reduce low-frequency drift and physiological high-frequency respiratory and cardiac noise. The details of ALFF calculation were reported in a previous study. ${ }^{41}$ To reduce the global effects of variability across the participants, the ALFF of each voxel was divided by the global mean value for each participant.

\section{rsFC differences}

After pre-processing, temporal filtering settings were applied using a band-pass filtered $(0.01 \sim 0.08 \mathrm{~Hz})$ to reduce lowfrequency drift and physiological high frequency respiratory and cardiac noise. The influence of covariant (including head motion parameters, global mean signal, white matter signal and cerebrospinal fluid signal) should be eliminated. The different areas from ALFF method and ICA method were selected as ROIs for rsFC analysis. The average time series from the ROIs were extracted from the residual image. To make the data fit the normal distribution, we calculated the coefficient of Pearson correlation between ROIs and other voxels of whole brain, the coefficient was participated in Fisher's Z transformation With Z value represents function connection coefficient. To reduce the global effects of variability across the participants, the rsFC of each voxel was divided by the global mean value for each participant.

\section{Receiver operating characteristic curve}

Receiver operating characteristic (ROC) curve analysis is a widely accepted method for identifying and comparing the diagnostic accuracy of biomarkers. Our previous study found that the ALFF method showed high sensitivity and specificity and may be a useful noninvasive imaging tool and an early biomarker for the detection of cerebral changes of obstructive sleep apnea patients. ${ }^{42}$ In this study, the ROC curve was used to investigate whether these specific ALFF differences have the sensitivity and specificity to distinguish SD status from RW status.

\section{Statistics}

The behavioral deficits were evaluated using two paired $t$-tests with the IBM Statistical Package for the Social Sciences version 21.0 (SPSS 21.0), and the statistical threshold was set at $P<0.05$.

For each selected component, individual maps were entered into one sample $t$-test $(P<0.0001$ with a minimum continuous cluster volume, $V$, of $1,080 \mathrm{~mm}^{3}$, corrected by false discovery rate). To reveal the between-condition differences of regional activities and connectivity patterns, paired $t$-tests were performed with Statistical Parametric Mapping 8 (SPM8, http://www.fil.ion.ucl.ac.uk/spm), with age and years of education as nuisance covariates of no interest. A corrected significance level of individual voxel $P<0.005$ and a continuous $V \geq 1,080 \mathrm{~mm}^{3}$, using an AlphaSim-corrected cluster threshold of $P<0.05$, were used to determine statistical significance.

\section{Results}

\section{Behavioral results}

Compared with RW subjects, SD subjects showed a lower response accuracy rate (RW mean accuracy rate $=96.83 \%$, $\mathrm{SD}$ mean accuracy rate $=77.67 \% ; t=-5.123 ; P<0.001$ ), a slower response (RW mean RT $=695.92 \mathrm{~ms}$; SD mean $\mathrm{RT}=799.18 \mathrm{~ms} ; t=3.892 ; P=0.003)$, a significantly higher lapse rate $(\mathrm{RW}$ mean lapse rate $=0.69 \%$, SD mean lapse rate $=19.29 \% ; t=5.762 ; P<0.001$ ), and a higher intraindividual coefficient of variability in $\mathrm{RT}$ ( $\mathrm{RW}$ coefficient $=0.26$, $\mathrm{SD}$ coefficient $=0.33 ; t=2.736 ; P=0.021$ ). The details are presented in Figure 1.

\section{DMN maps determined by group independent component analysis}

Figure 2 shows the DMN maps in the RW group and in the SD group respectively, as determined by the group independent 

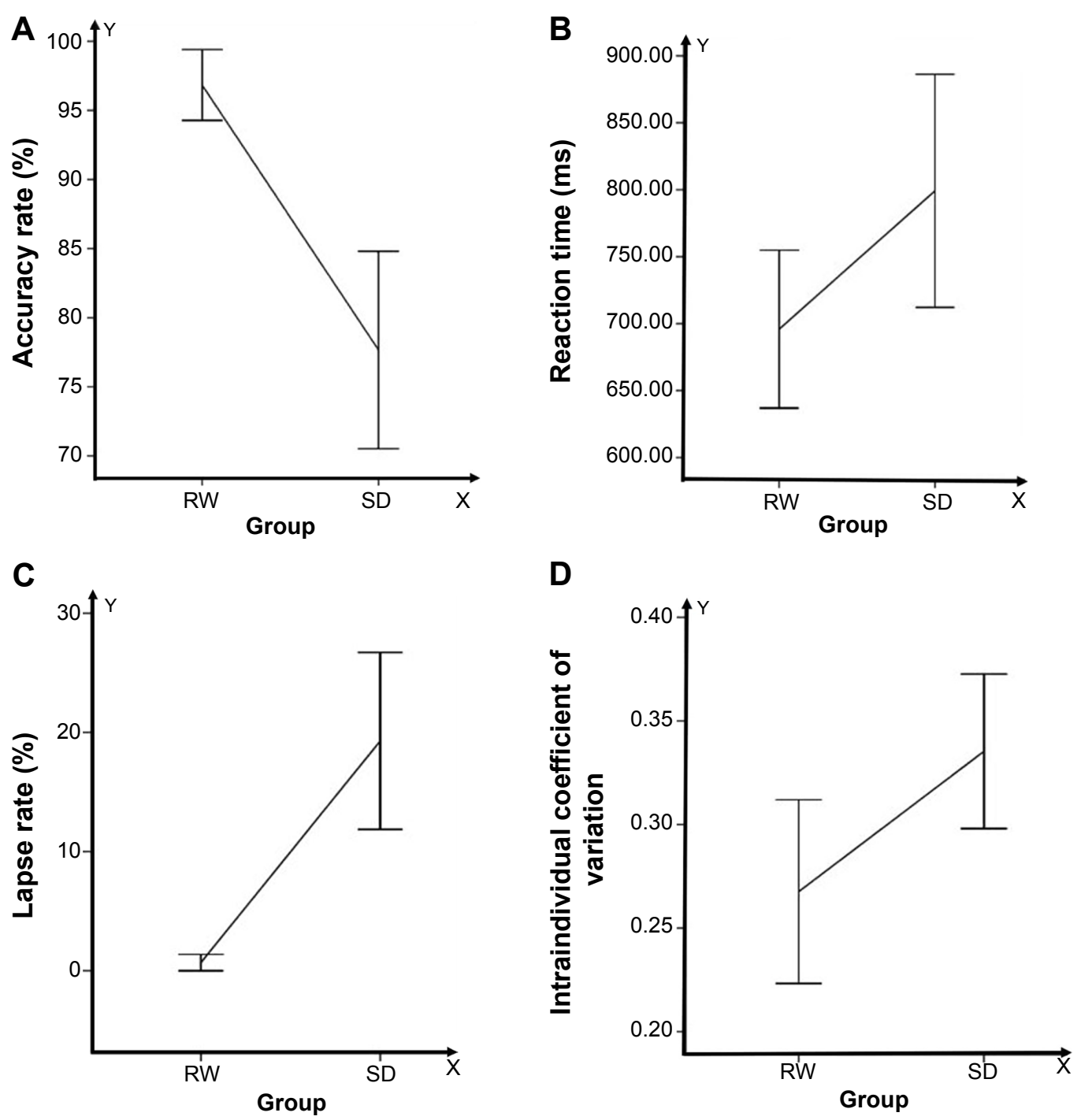

Figure I ANT findings of RW subjects and SD subjects.

Notes: Compared with RW subjects, SD subjects showed (A) lower accuracy rate, and (B) higher RT, (C) lapse rates, and (D) intraindividual variability. Abbreviations: ANT, attention network test; RW, rested wakefulness; SD, sleep deprivation; RT, reaction time.

component analysis (GICA) approach when performing the one-sample $t$-test. The areas within the DMN in the SD group were significantly smaller than those in the RW group.

The GICA method showed that SD subjects, compared with RW subjects, displayed decreased rsFC between whole brain region and right IPL (BA40) or left PrC/PCC (BA30, 31). The details are presented in Figure 3 and Table 1. The two different areas were selected as ROIs, namely, the IPL1-FC and PrC/PCC-FC maps, respectively.

\section{ALFF differences within the DMN}

Compared with RW subjects, SD subjects showed lower ALFF area in the left IPL (angular gyrus, supramarginal gyrus; BA39, 40). To further understand the intrinsic DMN impairment, a different region was selected as ROI for the subsequent rsFC analysis, namely, the IPL2-FC map. The details are presented in Figure 4 and Table 1.

\section{ROC curve}

Since the lower ALFF area was found in the left IPL in the $\mathrm{SD}$ group relative to the RW group, the brain region might be utilized as a marker to distinguish the SD status from the RW status. To test this possibility, the mean ALFF signal value of the region was extracted. The ROC curve analysis showed that the area under the curve (AUC) value of the left IPL was 0.75 , with a cutoff point of 0.834 (mean ALFF signal value). Further diagnostic analysis showed that the left IPL alone discriminated the SD status from the RW status with $75 \%$ sensitivity and $91.7 \%$ specificity. The details are presented in Figure 5. 

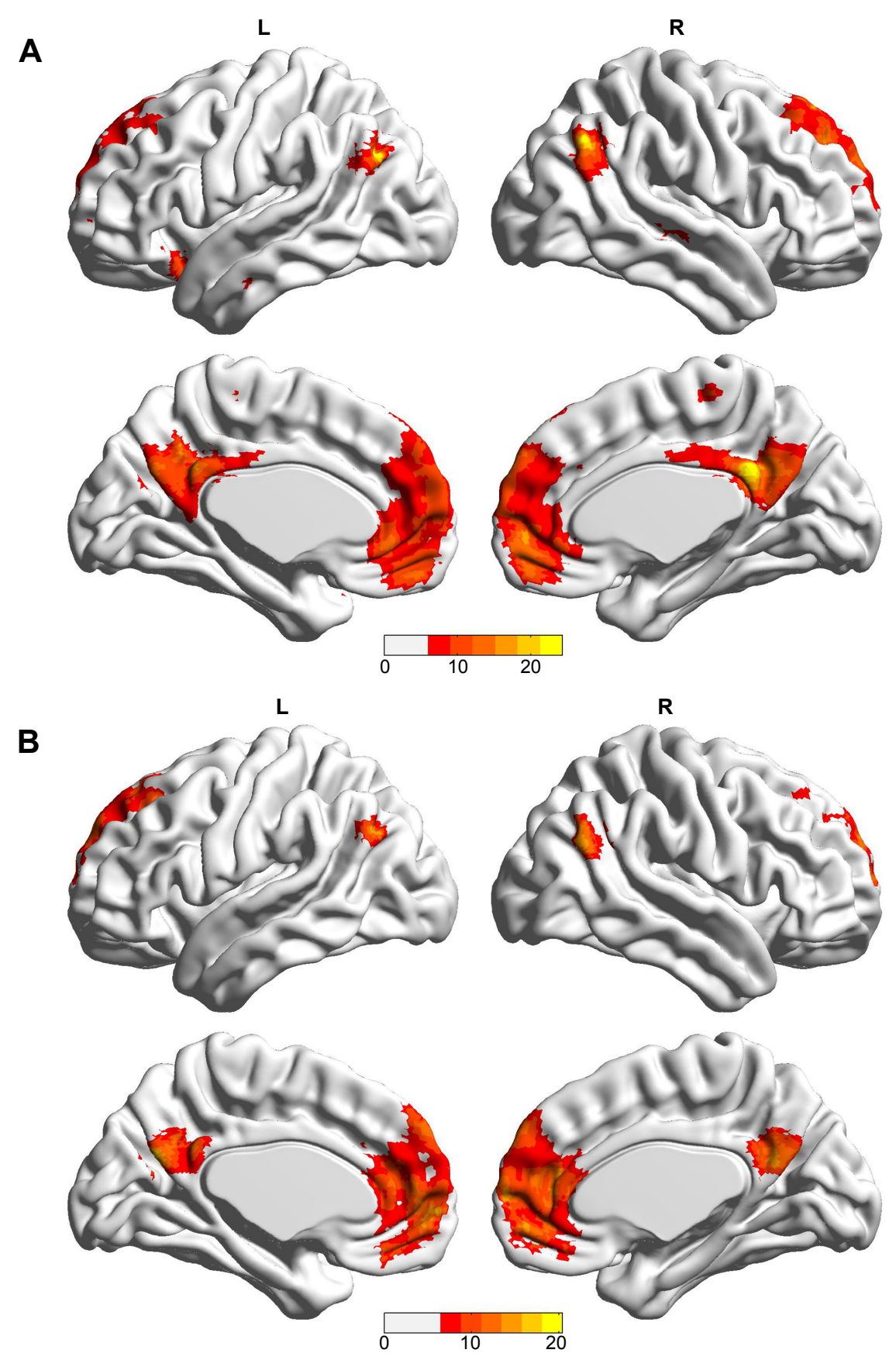

Figure 2 The DMN maps in (A) the RW and (B) the SD groups respectively.

Notes: The covered DMN areas were smaller in the SD group than in the RW group. These maps are the results of the within-group comparisons by the one-sample $t$-test with the GICA method, corrected by FDR.

Abbreviations: DMN, default-mode network; RW, rested wakefulness; SD, sleep deprivation; GICA, group independent component analysis; FDR, false discovery rate; L, left; R, right.

\section{Differences in rsFC}

The IPL1-FC map demonstrated that the right IPL showed decreased rsFC with the left Prc (BA7), in addition to increased rsFC with left fusiform gyrus (BA37) and left cluster of middle temporal gyrus and inferior temporal gyrus (BA37). The details are presented in Figure 3 and Table 1. No connectivity difference was found in the PrC/PCC-FC map. The IPL2-FC map showed that the left IPL had decreased $\mathrm{rsFC}$ with right cluster of IPL and superior temporal gyrus (BA39, 40). The details are presented in Figure 6 and Table 1.

\section{Discussion}

Our previous study had demonstrated that a few brain areas had obvious sex differences in both RW and SD subjects. ${ }^{3}$ 
A
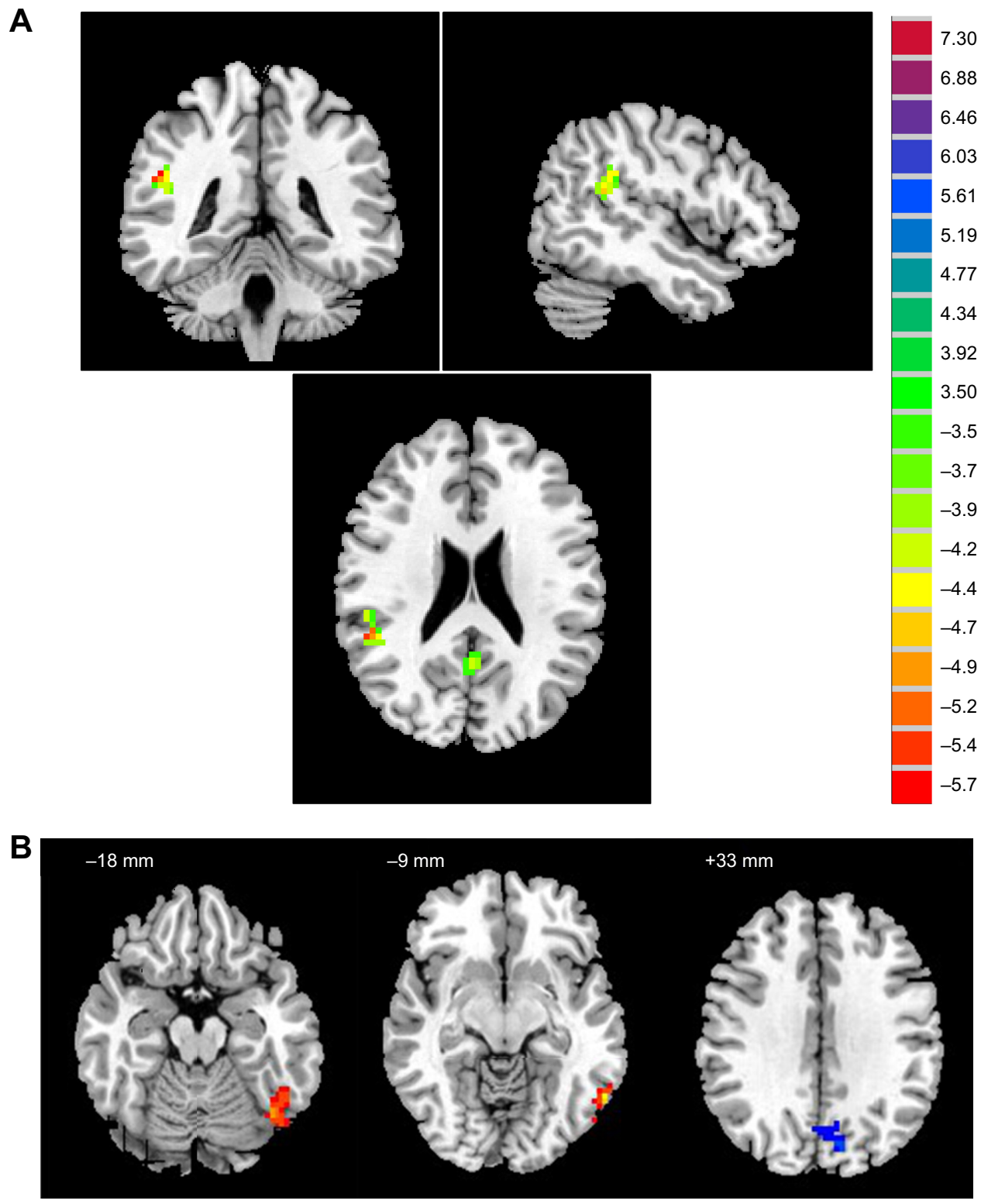

Figure 3 The rsFC differences between the SD group and the RW group.

Notes: (A) The three maps represent the results of the ICA method, which showed decreased rsFC in right IPL and left PrC/PCC in the SD group relative to the RW group. (B) The three maps reflected the result of the IPLI-FC map, which showed decreased rsFC between the right IPL and left Prc and increased rsFC between the right IPL and left fusiform gyrus and left middle/inferior temporal gyrus.

Abbreviations: rsFC, resting-state functional connectivity; SD, sleep deprivation; RW, rested wakefulness; ICA, independent component analysis; IPL, inferior parietal lobule; PrC, precuneus; PCC, posterior cingulate cortex; L, left; R, right.

To avoid the influence of sex differences, only healthy males were recruited in this study. This study demonstrated that both rsFC method and ALFF method could reflect the intrinsic DMN impairment, and both showed equivalent results. SD resulted in a decline in response accuracy rate, as well as significant increase in RT and lapse rate. Furthermore, compared with RW subjects, SD subjects showed significantly higher value of intraindividual coefficient of variability of RT. Sleep debt had been shown to reportedly augment emotional instability (eg, anxiety and confusion), dilatation of the pupils, sleepiness, and feeling of fatigue, receding psychomotor performance, and changes in heart rate and blood pressure. ${ }^{43-49}$ In this study, SD significantly reduced all the behavioral measures and increased the risk of the variability, because of which SD may increase emotional instability and have a tendency to increase the risk of developing a disease.

ROC curve has been previously used to discriminate disease from health in subjects. ${ }^{42,50}$ The discrimination result is considered excellent for AUC values between 
Table I Two-sample paired t-test differences within the DMN areas between SD subjects and RW subjects

\begin{tabular}{|c|c|c|c|c|c|c|c|}
\hline Methods & Conditions & $\begin{array}{l}\text { Brain regions of peak } \\
\text { coordinates }\end{array}$ & $R / L$ & BA & $V\left(\mathrm{~mm}^{3}\right)$ & $\begin{array}{l}t \text {-score of } \\
\text { peak voxel }\end{array}$ & $\begin{array}{l}\text { MNI coordinates } \\
\text { of peak voxel } \\
(x, y, z)\end{array}$ \\
\hline \multirow[t]{2}{*}{$\overline{I C A}$} & $\mathrm{SD}<\mathrm{RW}$ & $\mathrm{PrC} / \mathrm{PCC}$ & L & 31 & 1,890 & -4.92 & $-12,-57,30$ \\
\hline & & IPL & $\mathrm{R}$ & 40 & ।,755 & -5.94 & $5 \mathrm{I},-42,27$ \\
\hline ALFF & $\mathrm{SD}<\mathrm{RW}$ & $\begin{array}{l}\text { IPL (angular gyrus, } \\
\text { supramarginal gyrus) }\end{array}$ & L & 39,40 & 2,592 & -6.81 & $-48,-57,39$ \\
\hline \multirow[t]{3}{*}{ IPLI-FC } & $\mathrm{SD}<\mathrm{RW}$ & Prc & L & 7 & 1,188 & -4.31 & $-12,-78,33$ \\
\hline & $\mathrm{SD}>\mathrm{RW}$ & Fusiform gyrus & L & 37 & 86 & 5.99 & $-42,-63,-18$ \\
\hline & $\mathrm{SD}>\mathrm{RW}$ & $\begin{array}{l}\text { Middle temporal gyrus, } \\
\text { inferior temporal gyrus }\end{array}$ & L & 37 & 43 & 7.10 & $-60,-60,-9$ \\
\hline IPL2-FC & $\mathrm{SD}<\mathrm{RW}$ & IPL, superior temporal gyrus & $\mathrm{R}$ & 39,40 & 2,484 & -12.12 & $60,-57,18$ \\
\hline
\end{tabular}

Notes: The between-condition statistical threshold was set at cluster size with $P<0.05$, voxel with $P<0.005$ ( $|t| \geq 4.437)$, and $V \geq I, 080$ mm ${ }^{3}$, corrected for AlphaSim.

Abbreviations: DMN, default-mode network; SD, sleep deprivation; RW, rested wakefulness; R, right; L, left; BA, Brodmann's area; $V$, volume; MNI, Montreal Neurological Institute; ICA, independent component analysis; PrC, precuneus; PCC, posterior cingulate cortex; IPL, inferior parietal lobule; ALFF, amplitude of low-frequency fluctuation; FC, functional connectivity.
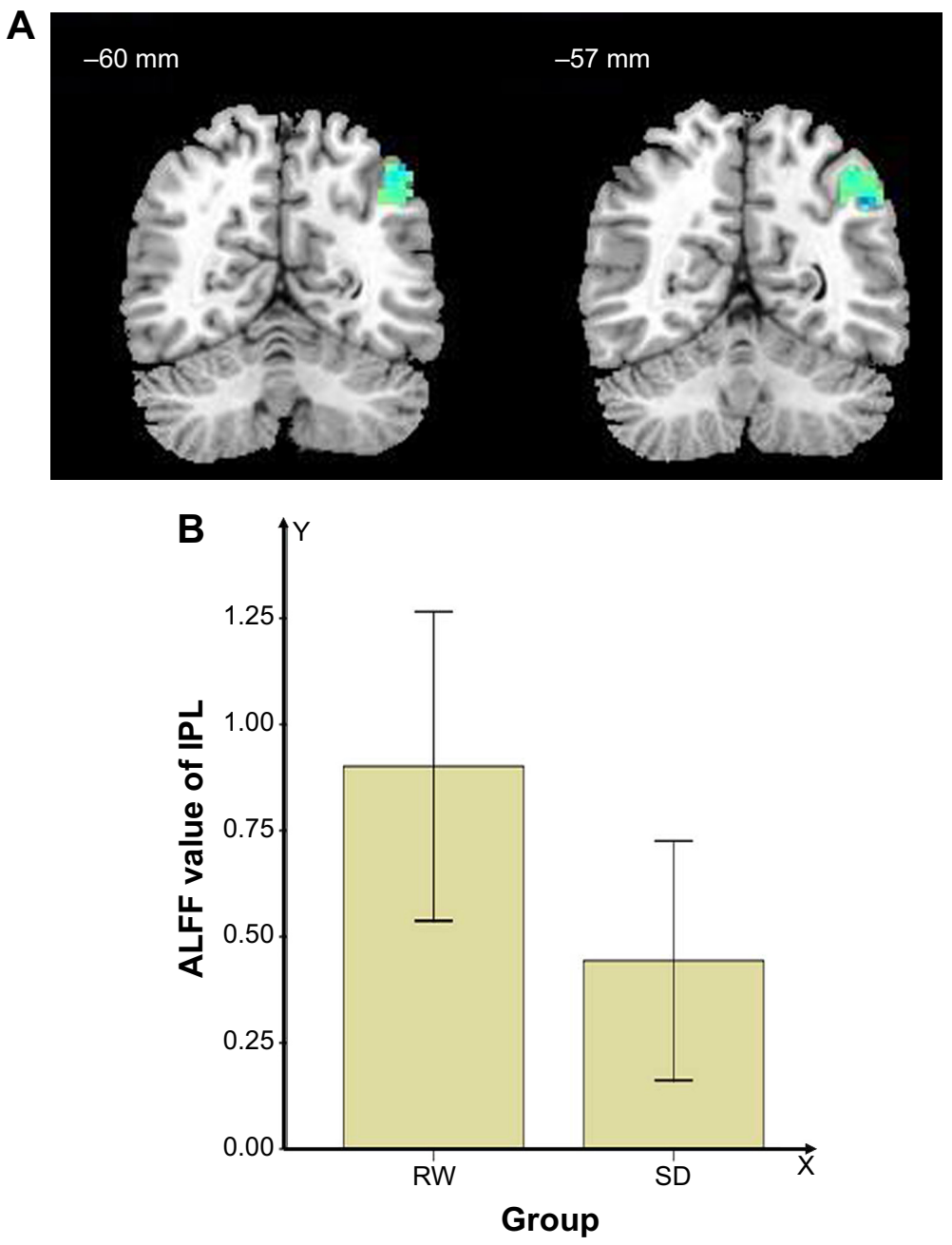

Figure 4 The ALFF differences within the DMN between the SD group and the RW group.

Notes: (A) Compared with RW group, SD group displayed lower ALFF area in the left IPL. (B) The right stapeldiagram presents the mean ALFF signal value of the left IPL area. The mean ALFF signal value of the left IPL was $0.90 \pm 0.57$ in the RW group, and that of the SD group was $0.44 \pm 0.44$, and the difference was statistically significant $(P=0.04)$.

Abbreviations: ALFF, amplitude of low-frequency fluctuation; DMN, default-mode network; RW, rested wakefulness; SD, sleep deprivation; IPL, inferior parietal lobule. 


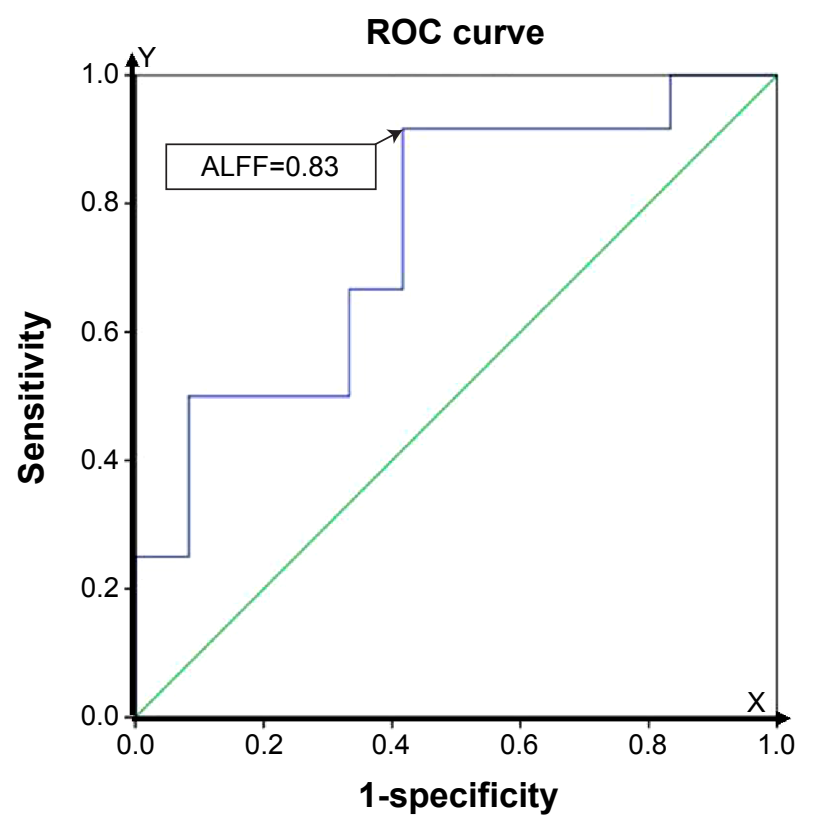

Figure 5 ROC curve of the mean ALFF value of the left IPL area.

Notes: The arrow represents the best cutoff point. The ALFF value represents the mean ALFF value of the corresponding time point.

Abbreviations: ROC, receiver operating characteristic; ALFF, amplitude of lowfrequency fluctuation; IPL, inferior parietal lobule.

0.9 and 1.0, good for values between 0.8 and 0.9 , fair for values between 0.7 and 0.8 , poor for AUC values between 0.6 and 0.7 , and failed for AUC values between 0.5 and $0.6 .^{51}$ In this study, ROC curve analysis revealed that the mean ALFF signal decline value in the left IPL had a fair AUC value (AUC $=0.75$ ). Further diagnostic analysis exhibited that it alone discriminated SD subjects from RW subjects, with $75 \%$ sensitivity and $91.7 \%$ specificity. In short, our findings suggest that the ALFF method might be an effective measurement method of the spontaneous neuronal activity in brain regions associated with the low-frequency BOLD fluctuations obtained by rfMRI, suggesting that the IPL might be applied as a trait-related node to identify SD status from RW status.

Interestingly, the GICA method showed that SD subjects, compared with RW subjects, displayed decreased rsFC between whole brain region and the right IPL or the left PrC/PCC. Furthermore, the right IPL area showed decreased rsFC with left Prc, heterolateral IPL, and small area in temporal lobe, as well as increased $\mathrm{rsFC}$ with left temporal lobe. These findings support intrinsic dysfunctional connectivity of the DMN, as shown by previous deep sleep and partial SD studies. ${ }^{23,24,52}$ The IPL region is obligatorily or unintentionally engaged in the recall, consolidation, and retrieval of episodic memory information, ${ }^{9,53,54}$ as well as being implicated in diverse cognitive operations ${ }^{55}$ that include bodily awareness, ${ }^{56}$ generating a sense of personal responsibility, ${ }^{57}$ and moral decision making. ${ }^{58}$ In this study, the IPL, as a core node, showed abnormal spontaneous activity and dysfunctional connectivity. We therefore speculate that the IPL might be an early and important hub node of default brain impairment and might be associated with abnormal emotional and cognitive symptoms.

$\operatorname{PrC}$ is a deactivated area with the highest metabolic rate during the resting state. ${ }^{9}$ Many diseases, such as obstructive sleep apnea, ${ }^{59}$ hyperactivity disorder, ${ }^{60}$ and amnestic type mild cognitive impairment, ${ }^{61}$ can lead to PrC impairment with the adjacent PCC being involved. The abnormally high metabolic rate and the distribution of arterial anatomy could explain the susceptibility of the PrC/PCC. Previous studies found that SD increased the RT and lapse rate, which was attributed to the less-efficient communication among brain areas $^{62,63}$ and to the changes in activity in the cingulate cortex. ${ }^{20,64}$ Sämann et a ${ }^{24,65}$ found that the PCC was associated with the regulation of consciousness, and the reduced integration of the PrC/PCC may be either an indicator of reduced awareness during wakefulness with increased sleep propensity or an indicator of sleep propensity itself. These findings suggest that the PrC/PCC may be involved in the worsening of behavioral measures (eg, RT, lapse rate) and sleep propensity by decreasing the rsFC among brain areas.

\section{Conclusion}

In this study, we demonstrated that long-term SD resulted in the intrinsic DMN dysfunction from both regional perspectives and integrative perspectives and that the DMN may be involved in the mechanism of cerebral and behavioral changes by decreasing efficient communication among brain areas. Furthermore, the IPL region might be an early and important default node of cerebral changes during SD status. Our findings provide insights into the pathophysiological mechanism of SD and may be helpful for understanding the pathophysiology of long-term SD and the development of symbolic early-imaging biomarkers for the detection of cerebral changes. ${ }^{66}$

\section{Acknowledgments}

This study was supported by the Jiangxi Provincial Department of Science and Technology Support Program (grant numbers 20121BBG70056, 20132BBG70061, and 20141BBG70026) and the Jiangxi Provincial Department of Natural Science Foundation Project (grant number 20132BAB205100) and National Basic Research Program of China (2011CB711000 and 2010SY5411003). 


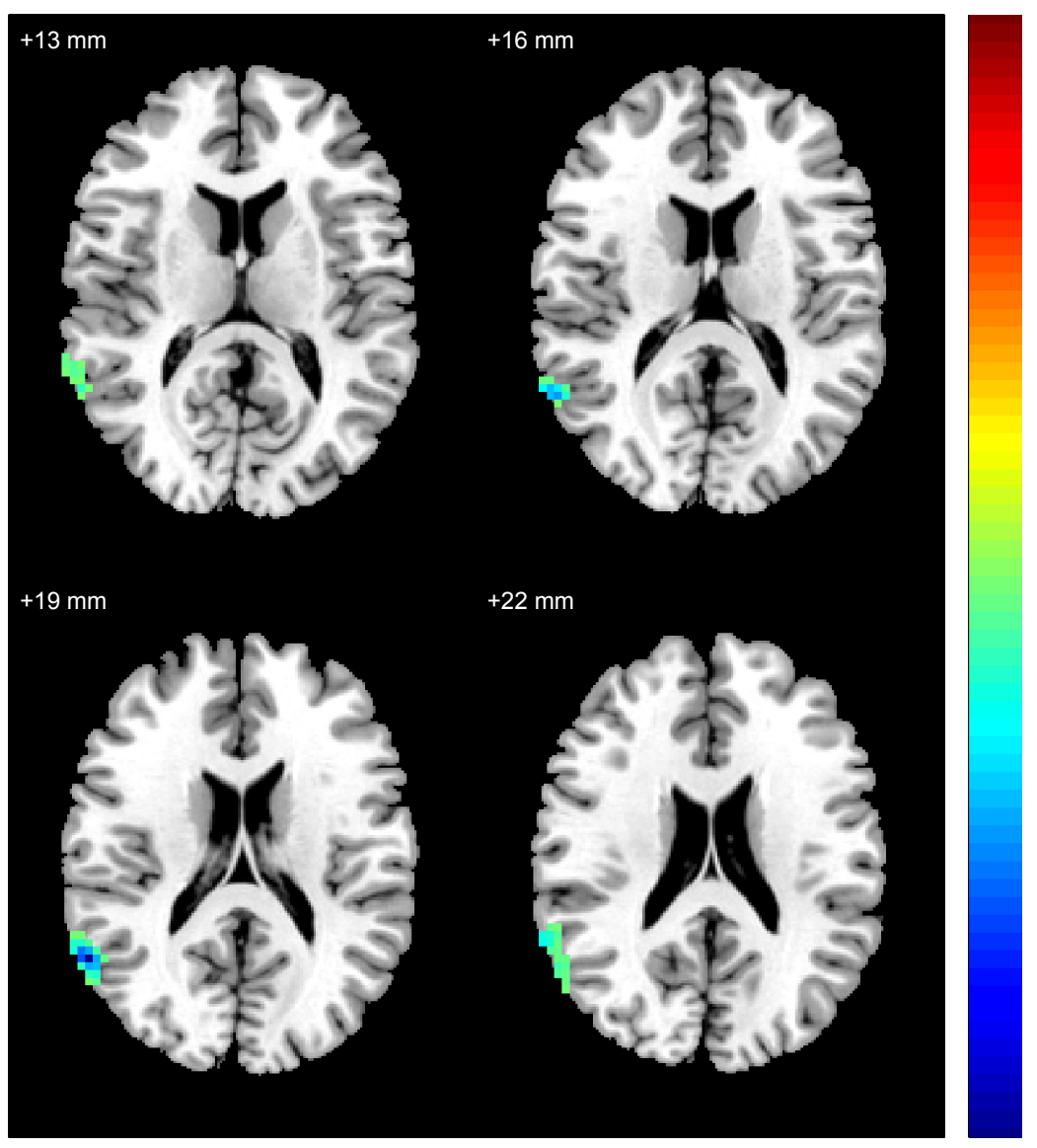

Figure 6 The IPL2-FC differences between SD and RW groups.

Notes: The color in the map represents the differences. The left IPL showed decreased rsFC with the right cluster of IPL and superior temporal gyrus $(P<0.005$, corrected for AlphaSim).

Abbreviations: IPL, inferior parietal lobule; FC, functional connectivity; SD, sleep deprivation; RW, rested wakefulness; rsFC, resting-state functional connectivity.

\section{Disclosure}

The authors report no conflicts of interest in this work.

\section{References}

1. Reimund E. The free-radical flux theory of sleep. Med Hypotheses. 1994; 43(4):231-233

2. Süer C, Dolu N, Artis AS, Sahin L, Yilmaz A, Cetin A. The effects of longterm sleep deprivation on the long-term potentiation in the dentate gyrus and brain oxidation status in rats. Neurosci Res. 2011;70(1):71-77.

3. Dai XJ, Gong HH, Wang YX, et al. Gender differences in brain regional homogeneity of healthy subjects after normal sleep and after sleep deprivation: a resting-state fMRI study. Sleep Med. 2012;13(6): 720-727.

4. Ohayon MM, Smolensky MH, Roth T. Consequences of shiftworking on sleep duration, sleepiness, and sleep attacks. Chronobiol Int. 2010; 27(3):575-589.

5. Tsigos C, Chrousos GP. Hypothalamic-pituitary-adrenal axis, neuroendocrine factors and stress. J Psychosom Res. 2002;53(4):865-871.

6. Walker MP, Stickgold R. Sleep, memory and plasticity. Annu Rev Psychol. 2006;57:139-166.

7. Buckner RL, Andrews-Hanna JR, Schacter DL. The brain's default network anatomy, function, and relevance to disease. Ann NY Acad Sci. 2008; 1124:1-38

8. Fox MD, Raichle ME. Spontaneous fluctuations in brain activity observed with functional magnetic resonance imaging. Nat Rev Neurosci. 2007;8(9):700-711
9. Gusnard DA, Raichle ME, Raichle ME. Searching for a baseline: functional imaging and the resting human brain. Nat Rev Neurosci. 2001;2(10):685-694.

10. Gusnard DA, Akbudak E, Shulman GL, Raichle ME. Medial prefrontal cortex and self-referential mental activity: relation to a default mode of brain function. Proc Natl Acad Sci. 2001;98(7):4259-4264.

11. Cabeza R, Dolcos F, Graham R, Nyberg L. Similarities and differences in the neural correlates of episodic memory retrieval and working memory. Neuroimage. 2002;16(2):317-330.

12. Simpson JR Jr, Drevets WC, Snyder AZ, Gusnard DA, Raichle ME. Emotion-induced changes in human medial prefrontal cortex: II. During anticipatory anxiety. Proc Natl Acad Sci. 2001;98(2):688-693.

13. Simpson JR Jr, Snyder AZ, Gusnard DA, Raichle ME. Emotion-induced changes in human medial prefrontal cortex: I. During cognitive task performance. Proc Natl Acad Sci. 2001;98(2):683-687.

14. Mason MF, Norton MI, Van Horn JD, Wegner DM, Grafton ST, Macrae $\mathrm{CN}$. Wandering minds: the default network and stimulusindependent thought. Science. 2007;315(5810):393-395.

15. Yan H, Zhang Y, Chen H, Wang Y, Liu Y. Altered effective connectivity of the default mode network in resting-state amnestic type mild cognitive impairment. J Int Neuropsychol Soc. 2013;19(4): 400-409.

16. Chee MW, Chuah LY. Functional neuroimaging insights into how sleep and sleep deprivation affect memory and cognition. Curr Opin Neurol. 2008;21(4):417-423.

17. Baliki MN, Geha PY, Apkarian AV, Chialvo DR. Beyond feeling: chronic pain hurts the brain, disrupting the default-mode network dynamics. J Neurosci. 2008;28(6):1398-1403. 
18. Lin WC, Hsu TW, Chen CL, et al. Connectivity of default-mode network is associated with cerebral edema in hepatic encephalopathy. PLoS One. 2012;7(5):e36986.

19. Murdaugh DL, Shinkareva SV, Deshpande HR, Wang J, Pennick MR, Kana RK. Differential deactivation during mentalizing and classification of autism based on default mode network connectivity. PLoS One. 2012;7(11):e50064.

20. Gujar N, Yoo SS, Hu P, Walker MP. The unrested resting brain: sleep deprivation alters activity within the default-mode network. $J$ Cogn Neurosci. 2010;22(8):1637-1648.

21. Bosch OG, Rihm JS, Scheidegger M, et al. Sleep deprivation increases dorsal nexus connectivity to the dorsolateral prefrontal cortex in humans. Proc Natl Acad Sci. 2013;110(48):19597-19602.

22. Chee MW, Chuah YM. Functional neuroimaging and behavioral correlates of capacity decline in visual short-term memory after sleep deprivation. Proc Natl Acad Sci. 2007;104(22):9487-9492.

23. Horovitz SG, Braun AR, Carr WS, et al. Decoupling of the brain's default mode network during deep sleep. Proc Natl Acad Sci. 2009;106(27): 11376-11381.

24. Sämann PG, Tully C, Spoormaker VI, et al. Increased sleep pressure reduces resting state functional connectivity. MAGMA. 2010;23(5-6): 375-389.

25. De Havas JA, Parimal S, Soon CS, Chee MW. Sleep deprivation reduces default mode network connectivity and anti-correlation during rest and task performance. Neuroimage. 2012;59(2):1745-1751.

26. Lu H, Zuo Y, Gu H, et al. Synchronized delta oscillations correlate with the resting-state functional MRI signal. Proc Natl Acad Sci. 2007; 104(46): 18265-18269.

27. Turner JA, Chen H, Mathalon DH, et al. Reliability of the amplitude of low-frequency fluctuations in resting state fMRI in chronic schizophrenia. Psychiatry Res. 2012;201(3):253-255.

28. Zuo XN, Di Martino A, Kelly C, et al. The oscillating brain: complex and reliable. Neuroimage. 2010;49(2):1432-1445.

29. Fransson P. Spontaneous low-frequency BOLD signal fluctuations: an fMRI investigation of the resting-state default mode of brain function hypothesis. Hum Brain Mapp. 2005;26(1):15-29.

30. Horovitz SG, Fukunaga M, de Zwart JA, et al. Low frequency BOLD fluctuations during resting wakefulness and light sleep: a simultaneous EEG-fMRI study. Hum Brain Mapp. 2008;29(6):671-682.

31. Dai XJ, Peng DC, Gong HH, et al. Altered intrinsic regional brain spontaneous activity and subjective sleep quality in patients with chronic primary insomnia: a resting-state fMRI study. Neuropsychiatr Dis Treat. 2014;10:2163-2175.

32. Fan J, McCandliss BD, Fossella J, Flombaum JI, Posner MI, et al. The activation of attentional networks. Neuroimage. 2005;26(2):471-479.

33. Fan J, McCandliss BD, Sommer T, Raz A, Posner MI. Testing the efficiency and independence of attentional networks. J Cogn Neurosci. 2002;14(3):340-347.

34. Satterthwaite TD, Elliott MA, Gerraty RT, et al. An improved framework for confound regression and filtering for control of motion artifact in the preprocessing of resting-state functional connectivity data. Neuroimage. 2013;64:240-256.

35. Yan CG, Cheung B, Kelly C, et al. A comprehensive assessment of regional variation in the impact of head micromovements on functional connectomics. Neuroimage. 2013;76:183-201.

36. Fox MD, Snyder AZ, Vincent JL, Corbetta M, Van Essen DC, Raichle ME. The human brain is intrinsically organized into dynamic, anticorrelated functional networks. Proc Natl Acad Sci. 2005;102(27) 9673-9678.

37. Saad ZS, Gotts SJ, Murphy K, et al. Trouble at rest: how correlation patterns and group differences become distorted after global signal regression. Brain Connect. 2012;2(1):25-32.

38. Calhoun VD, Adali T, Pekar JJ. A method for comparing group fMRI data using independent component analysis: application to visual, motor and visuomotor tasks. Magn Reson Imaging. 2004;22(9):1181-1191.

39. Calhoun VD, Adali T, Pearlson GD, Pekar JJ. A method for making group inferences from functional MRI data using independent component analysis. Hum Brain Mapp. 2001;14(3):140-151.
40. Oser N, Hubacher M, Specht K, Datta AN, Weber P, Penner IK. Default mode network alterations during language task performance in children with benign epilepsy with centrotemporal spikes (BECTS). Epilepsy Behav. 2014;33:12-17.

41. Zang YF, He Y, Zhu CZ, et al. Altered baseline brain activity in children with ADHD revealed by resting-state functional MRI. Brain Dev. 2007;29(2):83-91

42. Li HJ, Dai XJ, Gong HH, Nie X, Zhang W, Peng DC. Aberrant spontaneous low-frequency brain activity in male patients with severe obstructive sleep apnea revealed by resting-state functional MRI. Neuropsychiatr Dis Treat. In Press 2015.

43. Banks S, Van Dongen HP, Maislin G, Dinges DF. Neurobehavioral dynamics following chronic sleep restriction: dose-response effects of one night for recovery. Sleep. 2010;33(8):1013-1026.

44. Belenky G, Wesensten NJ, Thorne DR, et al. Patterns of performance degradation and restoration during sleep restriction and subsequent recovery: a sleep dose-response study. J Sleep Res. 2003;12(1):1-12.

45. Franzen PL, Buysse DJ, Dahl RE, Thompson W, Siegle GJ. Sleep deprivation alters pupillary reactivity to emotional stimuli in healthy young adults. Biol Psychol. 2009;80(3):300-305.

46. Minkel JD, Banks S, Htaik O, et al. Sleep deprivation and stressors: evidence for elevated negative affect in response to mild stressors when sleep deprived. Emotion. 2012;12(5):1015-1020.

47. Van Dongen HP, Maislin G, Mullington JM, Dinges DF. The cumulative cost of additional wakefulness: dose-response effects on neurobehavioral functions and sleep physiology from chronic sleep restriction and total sleep deprivation. Sleep. 2003;26(2):117-126.

48. Zhong X, Hilton HJ, Gates GJ, et al. Increased sympathetic and decreased parasympathetic cardiovascular modulation in normal humans with acute sleep deprivation. J Appl Physiol. 2005;98(6): 2024-2032.

49. Zohar D, Tzischinsky O, Epstein R, Lavie P. The effects of sleep loss on medical residents' emotional reactions to work events: a cognitiveenergy model. Sleep. 2005;28(1):47-54.

50. Guo WB, Liu F, Xun GL, et al. Reversal alterations of amplitude of lowfrequency fluctuations in early and late onset, first-episode, drug-naive depression. Prog Neuro Psychopharmacol Biol Psychiatry. 2013;40: 153-159.

51. El Khouli RH, Macura KJ, Barker PB, Habba MR, Jacobs MA, Bluemke DA. Relationship of temporal resolution to diagnostic performance for dynamic contrast enhanced MRI of the breast. J Magn Reson Imaging. 2009;30(5):999-1004.

52. Motomura Y, Kitamura S, Oba K, et al. Sleep debt elicits negative emotional reaction through diminished amygdala-anterior cingulate functional connectivity. PLoS One. 2013;8(2):e56578.

53. Foster DJ, Wilson MA. Reverse replay of behavioural sequences in hippocampal place cells during the awake state. Nature. 2006;440(7084): 680-683.

54. Wig GS, Grafton ST, Demos KE, Wolford GL, Petersen SE, Kelley WM. Medial temporal lobe BOLD activity at rest predicts individual differences in memory ability in healthy young adults. Proc Natl Acad Sci. 2008;105(47):18555-18560.

55. Laird AR, Eickhoff SB, Li K, Robin DA, Glahn DC, Fox PT. Investigating the functional heterogeneity of the default mode network using coordinate-based meta-analytic modeling. J Neurosci. 2009;29(46): 14496-14505.

56. Felician O, Anton JL, Nazarian B, Roth M, Roll JP, Romaiguère P. Where is your shoulder? Neural correlates of localizing others' body parts. Neuropsychologia. 2009;47(8-9):1909-1916.

57. Schaich Borg J, Hynes C, Van Horn J, Grafton S, Sinnott-Armstrong W. Consequences, action, and intention as factors in moral judgments: an fMRI investigation. J Cogn Neurosci. 2006;18(5):803-817.

58. Raine A, Yang Y. Neural foundations tomoral reasoning and antisocial behavior. Soc Cogn Affect Neurosci. 2006;1(3):203-213.

59. Peng DC, Dai XJ, Gong HH, Li HJ, Nie X, Zhang W. Altered intrinsic regional brain activity in male patients with severe obstructive sleep apnea: a resting-state functional magnetic resonance imaging study. Neuropsychiatr Dis Treat. 2014;10:1819-1826. 
60. Castellanos FX, Margulies DS, Kelly C, et al. Cingulate-precuneus interactions: a new locus of dysfunction in adult attention-deficit/ hyperactivity disorder. Biol Psychiatry. 2008;63(3):332-337.

61. Bai F, Zhang Z, Yu H, et al. Default-mode network activity distinguishes amnestic type mild cognitive impairment from healthy aging: a combined structural and resting-state functional MRI study. Neurosci Lett. 2008;438(1):111-115.

62. Chee MW, Tan JC, Zheng H, et al. Lapsing during sleep deprivation is associated with distributed changes in brain activation. J Neurosci. 2008;28(21):5519-5528.

63. Sadaghiani S, Scheeringa R, Lehongre K, Morillon B, Giraud AL, Kleinschmidt A. Intrinsic connectivity networks, alpha oscillations, and tonic alertness: a simultaneous electroencephalography/functional magnetic resonance imaging study. J Neurosci. 2010;30(30): 10243-10250.
64. Tomasi D, Wang RL, Telang F, et al. Impairment of attentional networks after 1 night of sleep deprivation. Cereb Cortex. 2009; 19(1):233-240.

65. Sämann PG, Wehrle R, Hoehn D, et al. Development of the brain's default mode network from wakefulness to slow wave sleep. Cereb Cortex. 2011;21(9):2082-2093.

66. Wang YX, Ng CK. The impact of quantitative imaging in medicine and surgery: charting our course for the future. Quant Imaging Med Surg. 2011;1(1):1-3.

\section{Publish your work in this journal}

Neuropsychiatric Disease and Treatment is an international, peerreviewed journal of clinical therapeutics and pharmacology focusing on concise rapid reporting of clinical or pre-clinical studies on a range of neuropsychiatric and neurological disorders. This journal is indexed on PubMed Central, the 'PsycINFO' database and CAS, and is the official journal of The International Neuropsychiatric Association (INA). The manuscript management system is completely online and includes a very quick and fair peer-review system, which is all easy to use. Visit http://www.dovepress.com/testimonials.php to read real quotes from published authors.

Submit your manuscript here: http://www.dovepress.com/neuropsychiatric-disease-and-treatment-journal 